\title{
Atención odontológica a pacientes con enfermedad de Von Willebrand
}

\section{Odontologic attention for patients with von Willebrand Disease}

\author{
de Miranda Chaves Netto HD*, Monteiro Aarestrup F**, Olate S***, \\ de Albergaria-Barbosa JR****, Mazzonetto R*****, Miranda Chaves MGA******
}

\section{RESUMEN}

La enfermedad de Von Willebrand, coagulopatía causada por la deficiencia de un gen que modifica la capacidad del organismo de producir factores suficientes que genere la coagulación, tienen un protocolo de tratamiento poco difundido entre los cirujanos-dentistas. El profesional debe estar alerta en pacientes con hemorragias mucocutáneas repetidas, especialmente si son asociadas a un patrón familiar, e iniciar su tratamiento multidisciplinar con el hematólogo, quien debería programar la reposición de factores que el paciente necesita para el tratamiento odontológico. En este trabajo presentamos un caso clínico de un paciente con enfermedad de Von Willebrand y discutimos el manejo odontológico para este paciente.

Palabras clave: Enfermedad de Von Willebrand, factor Von Willebrand, coagulopatía.

\section{SUMMARY}

The von Willebrand disease is a coagulopathy caused by the deficiency of the gene that modifies the organism's ability to produce sufficient factors that promotes coagulation. What is yet unknown by dental surgeons are the means to treat these patients. This patient has au functional abilities, but a coagulation disturbs is observed. It's a normal patient, but he bleeds excessively. The dentist showed always be attentive to the patient's familiar and pathological background, so he can initiate the dental treatment in synergy with the hematologist; who wile program the necessity or not of factor reposition that the patient might need during the treatment. In this article we present a clinical case of von Willebrand disease and we discuss the management odontological of this patient.

Key words: von Willebrand disease, von Willebrand factor, coagulopaty.

Fecha de recepción: 16 de febrero de 2009.

Aceptado para publicación: 23 de febrero de 2009.

* $\quad$ Estudiante de Doctorado. División de Cirugía y Traumatología Bucal y Maxilofacial. Facultad de Odontología de Piracicaba, Universidad Estadual de Campinas. Brasil.

** Profesor Adjunto. Coordinador del Programa de Especialización en Odontología para Pacientes Especiales. Facultad de Odontología, Universidad Federal de Juiz de Fora, Brasil.

*** Profesor. Departamento de Odontología Integral. Facultad de Medicina. Universidad de La Frontera. Chile; y Estudiante de Doctorado. División de Cirugía y Traumatología Bucal y Maxilofacial. Facultad de Odontología de Piracicaba. Universidad Estadual de Campinas. Brasil.

**** Profesor Titular. División de Cirugía y Traumatología Bucal y Maxilofacial. Facultad de Odontología de Piracicaba. Universidad Estadual de Campinas, Brasil

****** Profesora Adjunta. Programa de Especialización en Odontología para Pacientes Especiales. Facultad de Odontología. Universidad Federal de Juiz de Fora. Brasil.

de Miranda Chaves Netto HD, Monteiro Aarestrup F, Olate S, de Albergaria-Barbosa JR, Mazzonetto R, Miranda Chaves MGA. Atención odontológica a pacientes con enfermedad de Von Willebrand. Av. Odontoestomatol 2010; 26 (3): 131-137. 


\section{INTRODUCCIÓN}

La Enfermedad de Von Willebrand (EVW), descrita originalmente como un disturbio hemorrágico asociado a la herencia autosómica dominante, es hoy conocida con diferentes variantes genéticas (1-3). La Enfermedad de Von Willebrand es una alteración hereditaria, que presenta anormalidades en el Factor de Von Willebrand (FvW) encontrado en el plasma, plaquetas, megacariocitos y células endoteliales que circulan en conjunto con el factor VIII $(2,4,5)$.

Las principales funciones del FvW son:

1. Mediar la interacción entre las plaquetas y el colágeno subendotelial.

2. Mediar la interacción plaqueta-plaqueta.

3. Actuar como transportador molecular del FVIII y estabilizar su actividad coagulante $(2,3,4)$.

Con una frecuencia estimada de 1 en cada 800 a 1.000 personas, se cree que la EVW es uno de los disturbios hemorrágicos más comunes en los seres humanos $(2,6,7)$.

La EVW presenta varios subtipos que van desde la presentación asintomática hasta formas graves de la enfermedad. De este modo, en las formas leves de la EVW el paciente presenta sangrado mucocutáneo, donde la principal alteración es en la hemostasia primaria. Han sido descritos más de 20 variantes, que poden ser agrupadas en dos categorías principales, de acuerdo con la cantidad y calidad del $\operatorname{FvW}(5,6,8)$ :

- Tipo 1: Asociadas a cantidad reducida de FvW. Autosómica dominante; corresponde al $75 \%$ de los casos y es relativamente leve.

- Tipo 2: Asociadas a defectos cualitativos en el FvW, representa cerca del $17 \%$ de los casos. El subtipo 2A es el más común; es autosómica dominante con ausencia de multímeros de alto peso molecular (APM) donde el FvW formado es anormal y asociado al sangrado de discreto ha moderado, por lo cual es mas graves que las de tipo 1. El subtipo 2B presenta aumento de la afinidad del FvW a la glicoproteína plaquetaria 1B además de una secundaria perdida de multímeros APM, siendo usualmente acompañado de trombocitopenia (Tabla 1).
- Tipo 3: Son asociadas a cantidad (deficiencia severa de FvW con deficiencia secundaria de FVIII en varios grados). Es autosómica recesiva con niveles extremadamente bajos de FvW presentando manifestaciones clínicas graves asociadas a delaciones genéticas y mutaciones de dislocación; es menos común ( $1 \%$ de los pacientes) y clínicamente mas grave $(4,9,10)$ (Tabla 1).

En la EVW, el sangrado es causado por problema con proteínas sanguíneas que están presentes en el paciente normal ayudando en la formación del coágulo y a la detención del sangrado. Las personas con EVW no poseen la cantidad del factor suficiente o bien ellos trabajan de forma anormal $(10,11)$. Los pacientes presentan sangrado espontáneo de las mucosas, sangrado excesivo de las heridas, menorragias y aumento del tiempo de sangrado (TS) con un recuento plaquetario normal. El nivel plasmático de FvW, determinado como actividad del cofactor de ristocetina, se presenta también disminuido $(2,6,8)$. Como el FvW estabiliza el factor VIII al ligarse a el, la deficiencia de FvW provoca una reducción secundaria en los niveles del factor VIII, lo que puede reflejarse a través de un aumento del TTP (Tiempo de tromboplastina parcial) $(7,10)$.

Se realiza el diagnóstico a través del tiempo de sangría (TS) y del TTP (prolongados), nivel de FvW por medido por ELISA, actividad del cofactor ristocetina, inducción de agregación plaquetaria por la ristocetina (RIPA), análisis de FvW $(4,7)$ y a través del análisis del DNA (Técnicas de Biología Molecular). La biología molecular ofrece el diagnóstico y la clasificación de los subtipos a través de la investigación de la mutación directamente en el DNA, pero no es un examen disponible en muchos centros de diagnóstico de enfermedades hemorrágicas.

\section{TABLA 1.- ESQUEMA REPRESENTATIVO DE LA CLASIFICACIÓN DE LA ENFERMEDAD DE VON WILLEBRAND}

\begin{tabular}{|lcc|}
\hline Tipo EvW & Factor vW & \% Pacientes \\
\hline Tipo 1 & FvW bajo & $70-80$ \\
\hline Tipo 2A, 2B & FvW no actúa & $15-30$ \\
\hline Tipo 3 & FvW muy bajo & Infrecuente y grave \\
\hline
\end{tabular}


En el acompañamiento de pacientes con coagulopatías es importante el tratamiento de las hemorragias agudas, cuidados perioperatorios y profilaxis de las hemorragias (11-13). El sangrado gingival espontáneo y crónico, asociado a una buena higiene oral sin evidencias de enfermedad periodontal y el desarrollo de enfermedades hemorrágicas que afectan la cavidad oral, son muy compatibles con enfermedades de tipo hemorrágicas. La educación dirigida a estos pacientes es fundamental para la reducción de la morbilidad y de la mortalidad, con énfasis en el higiene oral, así como también en la vacunación contra hepatitis A e B (2).

Estudios recientes muestran que la infusión continua de concentrado de VIIIf e vWf $\left(8 \mathrm{Y}^{\circledR}\right)$ en pacientes con enfermedad de von Willebrand tipo I es segura, previniendo hemorragias importantes durante los procedimientos quirúrgicos (3).

Varios medicamentos pueden utilizarse en profilaxis o terapias postoperatorias odontológicas. Dentro de ellos están el ácido tranexámico, en comprimidos, que son ablandados con suero fisiológico y colocados en la herida quirúrgica con ligera compresión, pudiéndose utilizar también como baño para la cavidad oral $(8,12,13)$. Desmopresina (DDAVP), es el fármaco de elección en la terapia profiláctica de los pacientes tipo 1 y 2; su presentación para inhalar tiene la ventaja de no necesitar de hospitalización como lo necesita la vía endovenosa y eleva el factor VIII, con efecto mantenido hasta por 48 horas. La vía intramuscular no esta indicada $(8,12,14-17)$.

El objetivo de este informe de caso clínico es evaluar la respuesta al tratamiento odontológico en un paciente portador de Enfermedad de Von Willebrand, en quien fue realizado cirugías odontológicas menores junto al uso de FvW como estrategia para el control de la coagulopatía.

\section{PRESENTACIÓN DEL CASO CLÍNICO}

Paciente de 37 años, sexo femenino, blanca, brasileña portadora de la Enfermedad de Von Willebrand, compareció a la "Faculdad de Odontología de la Universidad Federal de Juiz de Fora", en el curso de "Especialização em Odontologia para Pacientes Por- tadores de Necessidades Especiais", reportando dolores articulares y sangramiento gingival. Su historia médica relataba sangrado significativo cuando se produjo el cambio de dentición de decidua a permanente y en la etapa de monarquía. A los 12 años de edad presento hemorragia cuando se le realizo una exodoncia, necesitando posteriormente de hospitalización por 40 días. Se reveló a través del examen (TTP) la enfermedad de Von Willebrand. Describió también, que al someterse a un procedimiento de cesárea se necesito la transfusión de plaquetas, uso de factor VIII y DDAVP (estimulador de FvW). La historia familiar relato otros casos de esta enfermedad en su familia (Fig. 1).

En el examen físico la paciente se presentaba con coloración normal, hidratada, eupneica, con frecuencia cardíaca de 82 bpm, presión arterial de $120 \times 80$ $\mathrm{mm}$. $\mathrm{Hg}$. Al examen clínico se verifico la necesidad de remoción de restos radiculares de dientes permanentes, restauraciones con resinas fotopolimerizables, confección de coronas protésicas, tratamiento periodontal y confección de placa miorrelajante. Mediante interconsultas con el hematólogo, fueron solicitados los exámenes complementarios, que presentaron los siguientes resultados: TP: 14,1 s; TTP: 32 s; Plaquetas: $370.000 / \mathrm{mm}$. La paciente exhibió $13 \%$ de actividades de los factores de coagulación; Factor VIII - 36\%. Para el día de la cirugía la paciente fue sometida a transfusión con FvW a las 8,00 horas de la mañana y fue orientada para retornar al "Hemocentro" y verificar la necesidad de otra transfusión. Los procedimientos odontológicos fueron iniciados 2 horas después que la paciente recibió el FvW. Es importante destacar el hecho de que antes

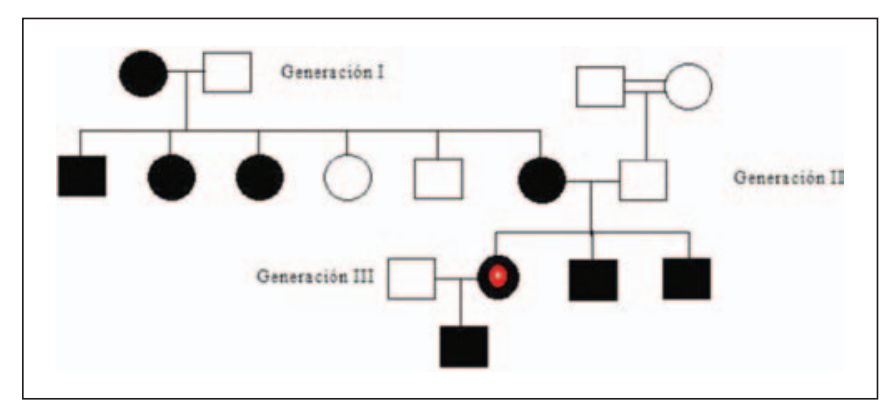

Fig. 1. Heredrograma de la paciente presentando múltiples casos familiares de Enfermedad de von Willebrand. La paciente corresponde a la III generación. 
de realizar la cirugía, debimos confirmar la disponibilidad del factor en la reserva del "Hemocentro", debido a la posible necesidad del paciente recibir otra dosis del factor.

En la primera fase del tratamiento se realizó la cirugía periodontal y las exodoncias de los restos radiculares, que correspondían a los procedimientos más invasivos dentro de los indicados para la paciente. Se realizó la anestesia infiltrativa de forma lenta, con un menor número de punciones posibles, utilizado el anestésico con vasoconstrictor de prilocaína al $3 \%$. La duración de los procedimientos fue de 45 minutos y durante la atención del paciente no ocurrió alteraciones de la presión arterial o de la frecuencia cardíaca. Los procedimientos quirúrgicos consistieron en la remoción de primer y segundo premolar derecho superior $(1,4$ y 1,5$)$ con caries extremadamente extensas y con compromiso periodontal que hacían inviable un tratamiento conservador; para ello después de la sindesmotomía fue realizado un colgajo mucoperiósticos, sin descarga, y solo con incisión sulcular vestibular para obtener mejor visibilidad de los dientes a extraer; las extracciones se realizaron con elevadores rectos y fórceps; finalmente se realizó una ostectomía para la regularización del reborde remanecente con fresa esférica de carbide montada en motor de baja rotación (25.000 rpm) y liberación del colgajo mucoperióstico, a través de incisiones en el periostio para permitir llegar a un cierre por primera intención. En el mismo tiempo quirúrgico fueron realizados alisados radiculares de elementos 1.7, que presentaba lesión de furca grado 2 y 1.3 para el cual se realizó cirugía periodontal con levantamiento de un colgajo sin descargas, con diagnostico de enfermedad periodontal avanzada. Las cirugías presentaron un transoperatorio y un posoperatorio satisfactorios con el sangrado considerado normal, gracias a una cirugía rápida y tranquila. Se realizó sutura compresiva con hilo cat-gut 3.0 junto a una esponja de fibrina en el alveolo; se efectuó la coaptación de los bordes de la herida para la cicatrización de primera intención y estabilización del coágulo. En las regiones de la cirugía periodontal y pulido radicular se utilizó cemento quirúrgico para la protección de la herida. No fue necesario, aunque a veces está indicado, irrigaciones para la cavidad bucal con ácido tranexámico (Transamim) diluido en suero fisiológico en la proporción de 1:10 de aplica- ción postquirúrgica; la última medida empleada fue la compresión con hielo en las primeras 24 horas. Se recomendó a la paciente no enjuagarse, evitando así la perdida del coágulo y del cemento quirúrgico.

La paciente relató discreto hematoma en el postoperatorio, el que fue también indoloro y sin presencia de infección. Después de siete días la paciente fue liberada por el hematólogo, para que se procediera a la eliminación de las suturas en algunas zonas que incomodaban a la paciente y también para la remoción del cemento quirúrgico. Las sesiones siguientes no necesitaron de los procedimientos hematológicos por ser consideradas atraumáticos.

\section{DISCUSIÓN}

Los pacientes portadores de la Enfermedad de Von Willebrand presentan disturbios de hemostasia, que llevan a presentar manifestaciones clínicas en diferentes grados, con signos y síntomas de intensidad variable. Para diferenciar la EVW de otras que exhiben manifestaciones semejantes, el diagnóstico esta basado en el principio de que esta enfermedad es el resultado de una mutación en el gen del factor Von Willebrand $(7,18)$.

Los análisis de laboratorio recomendados, consisten en la evaluación del hematocrito, hemoglobina, tiempo de protrombina, tiempo de tromboplastina y tiempo de sangrado. Además de estas, son sugeridas las dosis específicas del factor VIII, del antígeno del FvW y de la actividad del FvW $(9,10,19)$.

De la misma forma que un odontólogo realiza una consulta al médico frente a la atención de un paciente portador de disturbio hemorrágico, el médico también debería consultar al odontólogo a fin de discutir e integrar conceptos y estrategias sobre la conducta del tratamiento dentario respectivo $(4,9)$. Las exodoncias deben planificarse en base a las siguientes recomendaciones:

A. Excluir la presencia de anticuerpo inhibidor de factor antes de la cirugía.

B. Prevenir al paciente en cuanto al uso de medicaciones antiplaquetarias antes y después de la cirugía. 
C. Realizar la cirugía al iniciar el día y en el inicio de la semana, evitando y previniendo algunos problemas administrativos.

D. Certificar la existencia en cantidad suficiente de productos de reposición para el preoperatorio y postoperatorio, así como la reserva de sangre del fenotipo ya sometido a evaluación para enfermedades transmisibles por hemoderivados.

Se administra infusión del factor de coagulación con un tiempo mínimo de 20 minutos antes de la intervención. Este factor es prescrito por el hematólogo y entregado gratuitamente por el "Governo Federal do Estado de Minas Gerais do Brasil". De acuerdo con el protocolo de Agencia Nacional de Vigilancia Sanitaria Brasilera (ANVISA), se debe hacer aplicación previa de factor en el día de la extracción. En el caso de exodoncia, el paciente debe estar con el nivel plasmático de FvW de $10 \mathrm{mg} / \mathrm{l}$, por un mínimo de 5 días, dado que una discreta reducción en la concentración plasmática de FvW, o la pérdida selectiva de multímeros de alto peso molecular reduce la adhesividad plaquetaria, causando hemorragia clínica. En cirugías mas complicadas, además de la aplicación del factor se usa sellante o "cola" de fibrina, adaptándola en el interior del alvéolo y realizándose posteriormente sutura compresiva. La cola de fibrina es utilizada para mimetizar la fase final de la coagulación $(9,20)$.

Las orientaciones postoperatorias para el paciente incluyen: a) evitar el calor, b) ingerir alimentos líquidos y pastosos y c) no realizar ejercicios físicos, ayudando con esto a mantener el coágulo hasta que se desarrolle la cicatrización normal. Los planos quirúrgicos deben ser trazados de modo que permita una óptima hemostasia (4).

Baños locales con ácido tranexámico (Transamim) diluido en suero fisiológico en proporción de 1:10 deben ser considerados en los casos de intervenciones quirúrgicas y/o periodontales. En todos estos pacientes el uso de la aspirina y todos los medicamentos que contengan ácido acetil salicílico, así como también los antiinflamatorios no esteroidales (AINES), están rigurosamente contraindicados, porque afectan la función plaquetária $(4,8,12,13,17,18)$.

Se pueden realizar restauraciones simples y atraumáticas normalmente. La anestesia troncular sola- mente se hace con aplicaciones previa del factor debido a la aparición de hematomas.

En cuanto al aislamiento en los procedimientos restauradores, se debe dar preferencia al de tipo relativo, debido a la compresión del clamp sobre la mucosa. Se debe evitar aspiradores muy potentes en el piso de boca para no causar hematomas. La elección de las cubetas debe ser realizada con criterio para no herir la encía del paciente y deben ser protegidas con cera para evitar, precisamente, lesiones de la mucosa oral; debe ser cuidadosa en la formación de vacío, principalmente en el paladar blando, durante la toma de impresiones.

Existen medicamentos hemostáticos de acción sistémica que pueden utilizarse por los cirujanos-dentistas en el auxilio de cirugías. Dentro de ellos podemos citar la vitamina $K$, que estimula la síntesis de factores II, VII, IX y X. los antifibrinolíticos son indicados cuando ocurre aumento de los tiempos de protrombina y tromboplastina parcial para el control de la fibrinólisis. El ácido tranexámico tiene su indicación principalmente en uso local como compresivo o en baños bucales. La desmopresina estimula la liberación de FvW de las células endoteliales, con una indicación profiláctica por su tiempo de acción que llega a 48 horas. Etamsilato, que promueve disminución de tiempo de sangramiento y de la permeabilidad capilar y mejorar la adhesividad plaquetaria. Por ser desprovisto de efectos colaterales es considerado el hemostático de primera elección en odontología. Los estrógenos actúan produciendo vasoconstricción y disminuyendo la permeabilidad capilar, obliterando vasos lesionados y facilitando la formación del coágulo (1, 11-14, 16, 17, 20, 21).

Se debe evitar aquellos fármacos que interfieran con la función plaquetaria, como los antiinflamatorios no hormonales, además de las cefalosporinas que podrían causar anormalidades transitorias en la coagulación (4).

\section{CONCLUSIÓN}

El paciente portador de enfermedad de Von Willebrand puede recibir tratamiento dentario ambulatorial siempre que sea correctamente medi- 
cado, en cuanto a los aspectos de coagulación sanguínea, y sea realizado un tratamiento multidisciplinario incluyendo al hematólogo.

\section{BIBLIOGRAFÍA}

1. Federici AB. Clinical diagnosis of von Willebrand disease. Haemophilia 2004 Oct;10(4):169-76.

2. Ziv O, Ragni MV. Bleeding manifestations in males with von Willebrand disease. Haemophilia 2004 Mar; 10(2):162-8

3. Saroute AN, Brandão CMA, Guedes MA, Cavalheiro Filho C, Pomerantzeff PM. Patient with von Willebrand disease undergoing mitral valve repair: a strategy for the control of the coagulopathy Arq Bras Cardiol. 2007 Jan; 88(1):e4-6.

4. Israels S, Schwetz N, Boyar R, McNicol A. Bleeding Disorders: characterization, dental considerations and management. J Can Dent Assoc 2006 Nov;72(9):827-7i.

5. Tosetto A, Castaman G, Rodeghiero F. Assessing bleeding in von Willebrand disease with bleeding score Blood Rev. 2007 Mar;21(2):89-97.

6. Rodeghiero F, Castaman G, Tosetto A, Batlle J, Baudo F, Cappelletti A, et al. The discriminant power of bleeding history for the diagnosis of von Willebrand disease type I: an international, multicenter study. J Thromb Haemost 2005 Dec; 3(12): 2619-26.

7. Lippi G, Franchini M, Salvagno GL, Montagnana M, Poli G, Guidi GC. Correlation between von Willebrand factor antigen, von Willebrand factor ristocetin cofactor activity and factor VIII activity in plasma. J Thromb Thrombolysis 2007 Sep 3; [Epub ahead of print].

8. Franchini $M$. The use of desmopressin as a hemostatic agent: a concise review. Am J Hematol 2007 Aug;82(8):731-5.

9. Stubbs M, Lloyd J. A protocol for the dental management of von Willebrand's disease, haemophilia A and haemophilia B. Aust Dent $\mathrm{J}$ 2001 Mar;46(1):37-40.

10. Federici AB, Castaman G, Thompson A, Berntorp E. Von Willebrand's disease: clinical management. Haemophilia 2006 Jul;12(Suppl 3):152-8.

11. Siegmund B, Richter H, Pollmann H. Von Willebrand disease within the colletive of haemophilic patients as reason for unexpected bleeding episodes. Haemophilia 2007 Jan;13(1): 21-5.

12. Franchini M, Rossetti G, Tagliaferri A, Pattacini C, Lorenz C, Del Dot L, et al. Dental procedures in adult patients with hereditary bleeding disorders: 10 years experience in three Italian Hemofhilia Centers. Haemophilia 2005 Sep;11 (5):504-9.

13. Coetzee MJ. The use of topical crushed tranexamic acid tablets to control bleeding after dental surgery and from skin ulcers in haemophilia. Haemophilia 2007 Jul;13(4): 4434.

14. Lethagen S. Haemostatic treatment in connection with surgery in patients with von Willebrand disease. Haemofhilia 1999;5(2):64-7.

15. Revel-Vilk S, Schmugge M, Carcao MD, Blanchette P, Rand ML, Blanchette VS. Desmopressim, (DDAVP) responsiveness in children with von Willebrand disease. J Pediatr Hematol Oncol 2003 Nov;25(11):874-9.

16. Castaman G, Federici AB, Bernardi M, Moroni B, Bertoncello K, Rodeghiero F. Factor VIII and von Willebrand factor changes after desmopressin and during pregnancy in type $2 \mathrm{M}$ von Willebrand disease Vicenza: a prospective study comparing patients with single $(\mathrm{R} 1205 \mathrm{H})$ and double (R1205H-M7401) defect. J Thromb Haemost 2006 Feb;4(2):357-60.

17. Khair K, Baker K, Mathias M, Burgess C, Liesner R. Intranasal desmopressin (Octim TM): a safe and efficacious treatment option for children with 
bleeding disorders. Haemophilia 2007 Sep;13 (5):548-51

18. Halfpenny W, Fraser JS, Adlam DM. Comparison of 2 hemostatic agents for the prevention of postextraction hemorrhage in patients on anticoagulants. Oral Surg Oral Med Oral Pathol Oral Radiol Endod 2001 Sep;92(3):257-9.

19. Mazurier C. Composition, Quality Control, and Labeling of Plasma-Derived Products for the Treatment of von Willebrand disease. Semin Thromb Hemost 2006 Jul;32(5):529-36.

20. Piot B, Sigaud-Fiks M, Huet P, Fressinaud E, Trossaërt $M$, Mercier, J. Management of dental extractions in patients with bleeding disorders. Oral Surg Oral Med Oral Pathol Oral Radiol Endod 2002 Mar;93(3):247-50.

21. Morimoto Y, Yoshioka A, Sugimoto M, Imai Y, Kirita T. Haemostatic management of intraoral bleeding in patients with von Willebrand disease. Oral Dis 2005 Jul;11(4):243-8.

\section{CORRESPONDENCIA}

Dr. Henrique Duque de Miranda Chaves Netto Rua Halfeld 828 sl 311 Juiz de Fora -MG, Brasil Telefono (32) 32158352

e-mal: hdn@fop.unicamp.br 